\title{
Evaluation of Cervical High-Grade Squamous Intraepithelial Lesions-Correlated Markers as Triage Strategy for Colposcopy After Co-Testing
}

This article was published in the following Dove Press journal: OncoTargets and Therapy

\author{
Xiao Huo ${ }^{1, *}$ \\ Hengzi Sun ${ }^{2,3, *}$ \\ Dongyan $\mathrm{Cao}^{3}$ \\ Jiaxin Yang $^{3}$ \\ Peng Peng ${ }^{3}$ \\ Linghua Kong ${ }^{3}$ \\ Fei Chen ${ }^{3}$ \\ Keng Shen ${ }^{3}$ \\ Shuhong $\mathrm{Li}^{2}$ \\ 'Center of Basic Medical Research, \\ Peking University Third Hospital Institute \\ of Medical Innovation and Research, \\ Beijing, People's Republic of China; \\ ${ }^{2}$ Department of Obstetrics and \\ Gynecology, Beijing Chao-Yang Hospital, \\ Capital Medical University, Beijing, \\ People's Republic of China; ${ }^{3}$ Department \\ of Obstetrics and Gynecology, Peking \\ Union Medical College Hospital, Chinese \\ Academy of Medical Sciences and Peking \\ Union Medical College, Beijing, People's \\ Republic of China
}

*These authors contributed equally to this work

Correspondence: Shuhong Li Department of Obstetrics and Gynecology, Beijing Chao-Yang Hospital, Capital Medical University, No. 8

GongTiNan Road, Beijing, People's

Republic of China

Tel +86-10-8523 1831

Email1544563468@qq.com

Keng Shen

Department of Obstetrics and

Gynecology, Peking Union Medical

College Hospital, No. I Shuaifuyuan,

Dongcheng District, Beijing, I00730,

People's Republic of China

Email shenkengpumc@।63.com
Background: Colposcopy was referred in cases with severe abnormalities in co-testing. Although p16/Ki67 dual staining reduced the referral rate, its sensitivity and specificity need to be enhanced.

Methods: The expressions of p16, Ki-67, SMAD3, YAP1, RELA were evaluated in the colposcopy referral population. The inclusion criteria included 30-60 years and diagnosed with HPV16/18-positive, other HR-HPV-positive with ASCUS, LSIL, AGC (atypical glandular cell) in co-testing. Colposcopies, endocervical curettages of cervical biopsies were also collected. Cases were excluded if there were no biopsies, if the interval between a cervical screening test and biopsies was more than 6 months, or if insufficient tissue was available as a formalin-fixed paraffin-embedded block. The pathology was independently reviewed by two pathologists. Discrepant interpretations were adjudicated by a third pathologist.

Results: In total, 1194 of 1273 cases who were referred to colposcopy were evaluated in the present study. The sensitivity and specificity of p16+ combined with Ki-67+ for predicting CIN2+ were $62.1 \%$ and $89.5 \%$, respectively. p16+ combined with YAP1+ and/or RELA+ provided a sensitivity and specificity of $70.9 \%$ and $89.5 \%$, respectively, while $72.8 \%$ and $86.4 \%$ were achieved by p16+ combined with YAP1+ and/or SMAD3+ and/or RELA+. In HPV16/18+ and LSIL subgroups, the sensitivity and specificity of p16+ combined with Ki$67+$ for predicting CIN2+ were $67.7 \%$ and $87.6 \%$, respectively, for the former group and $58.6 \%, 88.8 \%$, respectively, for the latter group. p16+, YAP1+/RELA+ showed a better performance for predicting CIN2+ with a better sensitivity and considerable specificity in the other HPV+ combined with ASCUS group than were achieved by p16+ combined with Ki$67+$ RELA+ and the combination of 16 and RELA/YAP1 also provided the Max AUC area.

Conclusion: Our study shows that RELA and the combination of p16 and RELA/YAP1 achieved better sensitivity and specificity for detecting morphologically CIN2+ lesions.

Keywords: cervical precancer, immunocytochemical strategy, morphological marker, colposcopy triage

\section{Introduction}

Cervical cancer is the second most commonly occurring gynecologic cancer with a high mortality rate worldwide. ${ }^{1}$ With effective cervical screening based on the detection of cervical precancer according to tests for high-risk (HR) human papilloma virus (HPV) and cytology, the incidence and mortality of cervical cancer have decreased in recent decades. ${ }^{2-4}$ At present, the American College of Obstetricians and Gynecologists (ACOG) is still recommending co-testing for HPV and cytology 
in females aged 30-65 years old because co-testing achieves a higher sensitivity and specificity in cervical screening. 5

According to advanced screening guidelines, colposcopy is referred for the abnormal results of HPV and cytology tests, such as HPV16/18-positivity, other HR-HPV-positivity with atypical squamous cells of undetermined significance (ASCUS), low-grade squamous intraepithelial lesions (LSIL, cervical intraepithelial neoplasia [CIN1]) or high-grade squamous intraepithelial lesions (HSIL, cervical intraepithelial neoplasia $[\mathrm{CIN} 2+])$. However, many studies found that although co-testing effectively improved the effectiveness of cervical cancer screening, more than $60 \%$ of patients who were referred to invasive colposcopy with HSIL are $\leq$ CIN I for pathological results. ${ }^{6-11}$ Therefore, better triage strategies for colposcopy should be investigated to improve the accuracy of diagnoses and reduce the referral rate of colposcopy.

In 2012, the Lower Anogenital Squamous Terminology (LAST) Standardization Project recommended using immunohistochemical staining (IHC) for p16 to improve consistency in the diagnosis of HSIL (CIN2+). ${ }^{12}$ Recently, dual-stain cytology for p16 and $\mathrm{Ki}-67$ has been proposed as a biomarker for colposcopy triage and to have a sensitivity ranging from $68 \%$ to $94.2 \%$ and a specificity ranging from $30.6 \%$ to $95.2 \%$ in different subgroups. ${ }^{13-16}$ A study based on the VUSA Screen compared genotyping results with the results of p16/Ki-67 dual-staining and reported that the sensitivity of $\mathrm{p} 16 / \mathrm{Ki}-$ 67 dual-stained cytology for the detection of CIN2+ tended to be higher than that of HPV16/18 genotyping (68.8\% vs $43.8 \%$ ). However, the specificity for detecting CIN2+ was significantly lower for p16/Ki-67 dual-staining than for HPV16/18 genotyping $(72.8 \%$ vs $79.4 \%) .{ }^{17}$ Another multi-center study analyzed 1357 patients and showed that sensitivity (92.7\% vs $94.5 \%)$ and specificity (52.7\% vs 53.5\%) were higher for p16/Ki67 double staining than for cytology in the HPV-positive group. Moreover, although p16/Ki-67 showed better specificity than the HR-HPV test $(66.4 \%$ vs $55.8 \%)$, the sensitivity of p16/Ki-67 was worse than that of the HR-HPV test $(87.5 \%$ vs $91.7 \%)$ in the ASCUS/LSIL group. ${ }^{18}$ The New 2019 Principles of ASCCP recommended that HPV-based testing is the basis for risk estimation. The term HPV-based testing is used throughout this document and refers to the use of either primary HPV testing alone or HPV testing in conjunction with cervical cytology (co- testing). Importantly, guidelines must allow updates to incorporate new test methods as they are validated, and to adjust for decreasing CIN3+ risks as more patients who received HPV vaccination reach screening age. ${ }^{19}$ Therefore, more disease-specific molecular markers of cervical high-grade squamous intraepithelial lesions should be investigated to provide a triage strategy with high sensitivity and high specificity for colposcopy after co-testing.

Recently, SMAD3, RELA and YAP1 have been demonstrated that they played major roles in sensing shape information of cells by systematically analyzing the interactions between shape-correlated genes and key EMT transcription factors. ${ }^{20}$ SMAD family member 3 (SMAD3), a signal transducer and transcriptional modulator that mediates multiple signaling pathways, is activated by transforming growth factor-beta (TGF- $\beta$ ) and thought to play a role in the regulation of carcinogenesis. Recent studies have shown that Twist induces epithelial-mesenchymal transition (EMT) by regulating the TGF- $\beta / \mathrm{Smad} 3$ signaling pathway in cervical carcinogenesis and that the activation of TGFA/Smad3 signaling can induce the migration and invasion of cervical cancer cell lines, suggesting that it plays a vital role in cervical cancer metastasis. $^{21,22}$ Yes-associated protein-1 (YAP1) encodes a downstream nuclear effector of the Hippo signaling pathway, which is involved in development, growth, repair, and homeostasis. This gene is known to play a role in the development and progression of multiple cancers by acting as a transcriptional regulator of this signaling pathway and may therefore function as a potential target for cancer treatment. Xiao et al showed that nuclear levels of YAP are higher in high-grade cervical lesions and that YAP can function as a predictive marker for cervical cancer. ${ }^{22}$ In addition, YAP was correlated with HPV infection and found to localize to the nucleus in HPV-positive squamous cell carcinoma. ${ }^{23}$ RELA proto-oncogene (RELA, NF-kB subunit, NF-kB 3) is composed of NF-kB1 complexed with the product of this gene is the most abundant form of NF-kappa-B. Studies have reported that the nuclear expression of NF$\mathrm{kB}$ might be considered an indicator of malignant transformation and that the deregulation of RELA is a characteristic common feature of cervical tumors during progression. ${ }^{24}$ Here, we evaluated the correlations among the expression of $\mathrm{p} 16, \mathrm{Ki}-67$, the shape-correlated genes SMAD3, YAP1, RELA and CIN2+ lesions using IHC in the colposcopy referral population. 


\section{Materials and Methods}

\section{Study Population}

A search was performed in the Peking Union Medical College Hospital and Beijing Chao-Yang Hospital database for data entered from January 1, 2015 to January 1, 2017 related to screening women who were $30-60$ years and diagnosed with HPV16/18-positive, other HR-HPVpositive with ASCUS, LSIL, AGC (atypical glandular cell) in co-testing. Subsequent colposcopies, endocervical curettages and cervical biopsies were also collected. Cases were excluded if there were no biopsies, if the interval between a cervical screening test and biopsies was more than 6 months, or if insufficient tissue was available as a formalin-fixed paraffin-embedded block.

\section{Immunohistochemistry}

All formalin-fixed paraffin-embedded blocks were obtained from the Department of Pathology, Peking Union Medical College Hospital and Beijing Chao-Yang Hospital. Hematoxylin and eosin staining (H\&E) had been previously performed on all cases at the time of original diagnosis. p16, Ki-67, SMAD3, YAP1 and RELA immunohistochemistry were performed in all included cases. Antibodies were purchased from Abcam (Cambridge, USA). The concentration of each primary antibody was set as follows: p16 (1:1000), Ki-67 (1:1000), SMAD3 (1:2000), YAP1 (1:1000) and RELA (1:2000). In addition, immunohistochemistry was conducted according to the manufacturer's protocol.

p16-positive staining was defined as continuously strong, diffuse, bank-like nuclear or nuclear plus cytoplasmic staining involving at least one third of the squamous epithelium thickness. Negative staining was defined as all other staining patterns, such as cytoplasmic only, wispy, blob-like, puddled, scattered, and single cells, according to the LAST guidelines. ${ }^{12}$ Positive staining for Ki-67, SMAD3, YAP1 and RELA was defined as follows: five random microscope fields were selected to evaluate semiquantification staining. The intensity of immunostaining was graded as $1+$, weak; $2+$, moderate; or $3+$, strong. The area of positive squamous epithelium cells in each microscopic field was categorized as $1+$ for $0 \%$ to $33 \% ; 2+$ for $33 \%$ to $66 \%$; or $3+$ for $66 \%$ to $100 \%$ coverage. A sum between 5 and 45 was obtained by multiplying the 2 scores by 5. A sum from 0 to 17 was defined as "low expression (-)", while a sum from 18 to 45 was defined as "high expression $(+)$ ". Examples of observed staining characteristics of different expressions are represented in Figure 1. Negative IHC scores for p16, Ki-67, SMAD3, YAP1 and RELA show as Figure 1A, C, E, G and I. Positive IHC scores for p16, Ki-67, SMAD3, YAP1 and RELA show as Figure 1B, D, F, H and J. No specific quantitative criteria were defined for the number of positive cells. Two pathologists independently reviewed the $\mathrm{H} \& \mathrm{E}$ and IHC slides using their individual criterion, and the consensus interpretations were used as the final interpretations. Discrepant interpretations were adjudicated by a third pathologist.

\section{Follow-Up}

All cases which showed NILM and CIN1 in cervical biopsies were informed to re-test by HPV genotyping and liquid-based cytology 12 months later, which lasting 3 years. The colposcopy and cervical biopsies were referred when the results of re-tests were in line with screening guidelines.

\section{Statistical Analysis}

The associations between p16, Ki-67, SMAD3, YAP1 and RELA positivity and subsequent CIN2+ were analyzed using Chi-squared Tests. The sensitivity, specificity, Youden's index and Area Under Curve (AUC) of each biomarker as a predictor for $\mathrm{CIN} 2+$ were determined, and the different assays were compared using the McNemar test. A P-value of $<0.5$ was considered significant.

\section{Results}

In total, 1273 patients with complete results for HPV genotyping and liquid-based cytology tests met the initial inclusion criteria. Of these 1273 patients, 79 were excluded for no available cervical biopsy results. Of the remaining 1194 patients, 106 underwent endocervical curettage, and 52 consisted of vaginal HPV and cytology tests followed by a vaginal biopsy. In total, 1194 cases were available for evaluation, including 534 diagnosed with no intraepithelial lesion or malignant lesion (NILM), 289 diagnosed with CIN1, 173 diagnosed with CIN2, 160 diagnosed with CIN3 and 38 diagnosed with cancer. Patient ages ranged from 30 to 60 years old, and the median age was 40 years old.

The 1194 cases comprising the HPV16/18+ cases $(\mathrm{n}=606)$, other HPV+ and ASCUS cases $(\mathrm{n}=339)$, LSIL cases $(\mathrm{n}=381$, including 105 cases with HPV16/18+ and 249 cases with other HPV+) were analyzed. The associations between HPV genotype, cytology and corresponding 

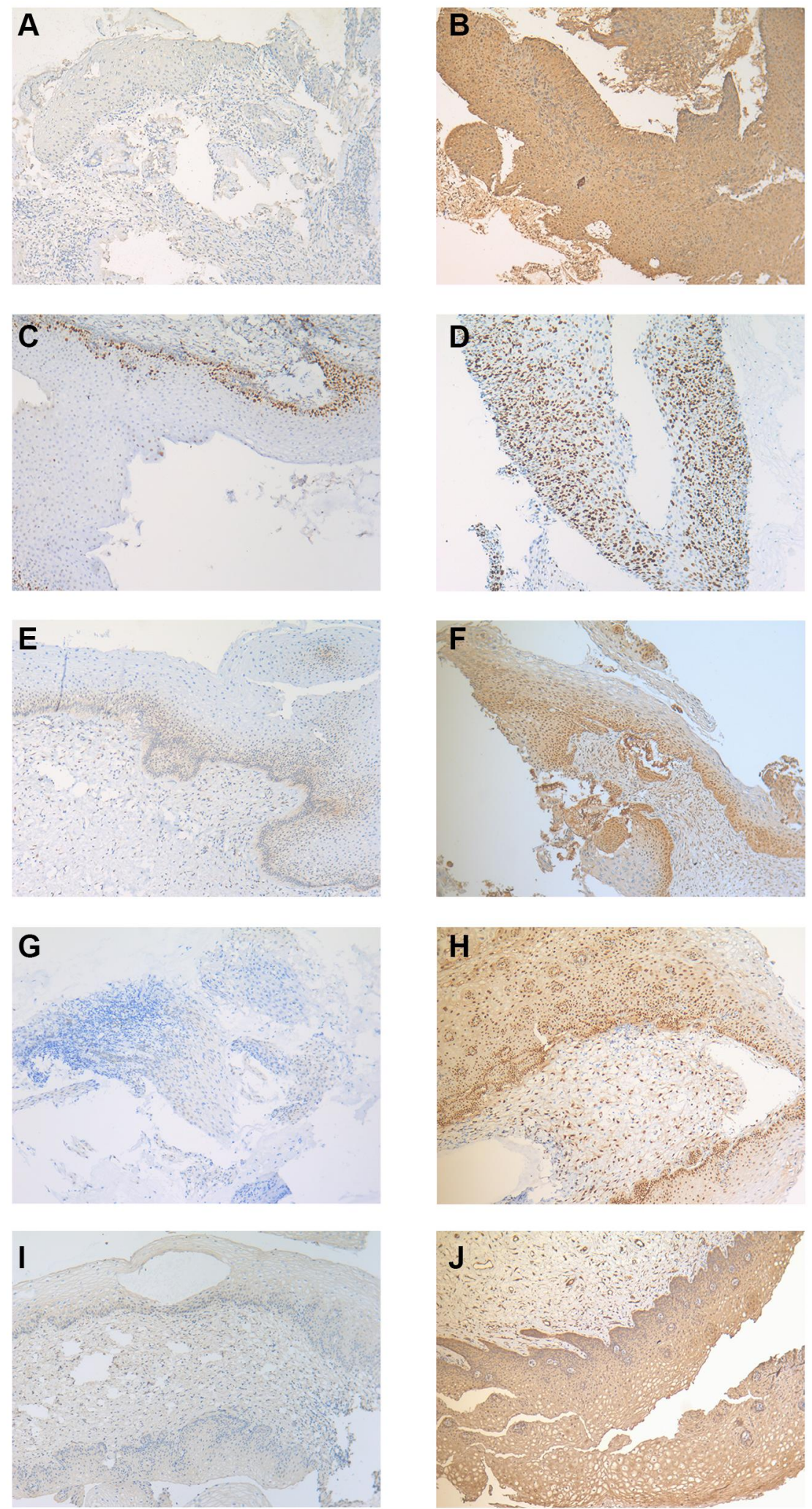

Figure I The staining characteristics of different expression pattern for pl6, Ki-67, SMAD3, YAPI and RELA (I00X). Negative IHC scores for pI6 (A), Ki-67 (C), SMAD3 (E), YAPI (G) and RELA (I). Positive IHC scores for p16 (B), Ki-67 (D), SMAD3 (F), YAPI (H) and RELA (J). 
Table I Association Between HPV Genotyping, Cytology, and Histology in the Enrolled Patients

\begin{tabular}{|c|c|c|c|c|c|c|c|}
\hline & \multicolumn{5}{|c|}{ Histology } & \multirow[t]{2}{*}{ Total (N) } & \multirow[t]{2}{*}{ Rate of CIN2+ (\%) } \\
\hline & NILM & CIN I & CIN 2 & CIN 3 & $\mathbf{C a}$ & & \\
\hline HPVI6/18+ & 318 & 93 & 78 & 87 & 30 & 606 & 32.2 \\
\hline HPVI6/I8+, NILM & 153 & 18 & 9 & 24 & 18 & 222 & 23.0 \\
\hline HPVI6/18+, ASCUS & 129 & 48 & 39 & 51 & 12 & 279 & 35.9 \\
\hline HPVI6/I8+, LSIL & 36 & 27 & 30 & 12 & 0 & 105 & 40.0 \\
\hline Other HPV+, ASCUS & 204 & 66 & 39 & 30 & 0 & 339 & 20.4 \\
\hline Other HPV+, LSIL & 117 & 87 & 21 & 24 & 0 & 249 & 18.1 \\
\hline LSIL & 153 & 114 & 51 & 36 & 0 & 354 & 24.6 \\
\hline Total (N) & 639 & 246 & 138 & $14 \mid$ & 30 & 1194 & 25.9 \\
\hline
\end{tabular}

histology are listed in Table 1. CIN2+ was confirmed in $32.2 \%(195 / 606)$ of HPV16/18+ cases. CIN2+ was confirmed in $20.4 \%(69 / 339)$ of other HPV+ and ASCUS specimens. CIN2+ was confirmed in $24.6 \%$ (87/354) of LSIL cases. Of the 1194 cases, 76 cases showed discrepant results among different IHC markers and were adjudicated by a third pathologist. Finally, 516 (43.2\%), 336 (28.1\%), 348 (29.1\%), $369(30.9 \%)$, and 255 (21.4\%) samples were positive for $\mathrm{p} 16$, Ki-67, RELA, YAP1 and SMAD3, respectively. The positive rates of p16, Ki-67, RELA, YAP1 and SMAD3 were also significantly different in biopsy $<\mathrm{CIN} 2$ and CIN2+ cases $(p<0.05$, Figure 2). The positive rate of each combination of p16, Ki-67, RELA, YAP1 and SMAD3 staining was also determined. There were 285 (23.9\%), 255 (21.4\%), 243 (20.4\%), 162 (13.6\%), $312(26.1 \%)$ and 345 (28.9\%) cases that were positive for p16 combined with Ki-67, p16 combined with RELA, p16 combined with YAP1, p16 combined with SMAD3, p16 combined with YAP1 or RELA, and p16 combined with YAP1 or SMAD3 or RELA, respectively (Table 2). The sensitivity and specificity of the combination of potential markers for predicting biopsy CIN2+ are also shown in Table 2. The sensitivity, specificity and

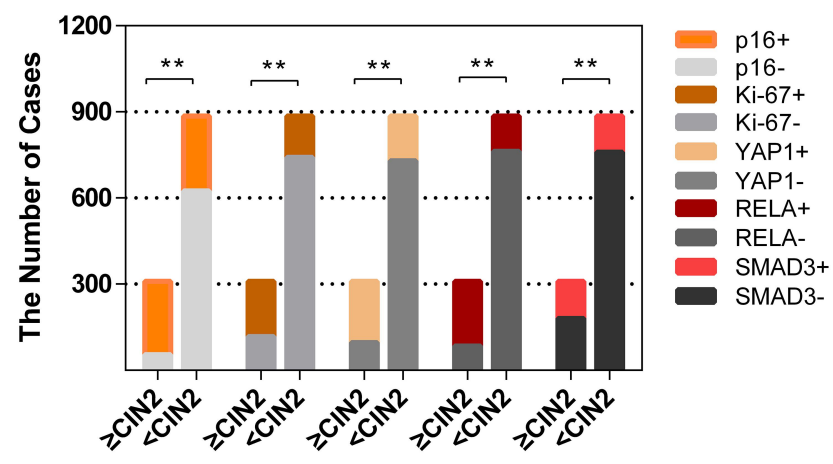

Figure 2 The positive rates of pl6, Ki-67, SMAD3, YAPI and RELA in biopsy $<\mathrm{CIN} 2$ and $\mathrm{CIN} 2+$, respectively $(* * p<0.0 \mathrm{I})$.
Youden's Index of p16 combined with $\mathrm{Ki}-67$ for predicting CIN2+ were $62.1 \%, 89.5 \%$ and $51.6 \%$, respectively. However, p16+ combined with YAP1+ and/or RELA+ (p16+, YAP1+/RELA + ) provided a better sensitivity $(70.9 \%$ vs $62.1 \%, \quad \mathrm{p}=0.184)$, considerable specificity $(89.5 \%$ vs $89.5 \%, p=1)$ and better Youden's Index $(60.4 \%$ vs $51.6 \%$ ). Compared with p16+ combined with Ki-67+. p16 + combined with YAP1+ and/or SMAD3+ and/or RELA+ (p16+, YAP1+/SMAD3+/RELA + ) showed a better sensitivity $(72.8 \%$ vs $62.1 \%, \mathrm{p}=0.102)$, considerable specificity $(86.4 \%$ vs $89.5 \%, \mathrm{p}=0.255)$ and better Youden's Index (59.2\% vs.51.6\%) compared with p16+ combined with $\mathrm{Ki}-67+$ in the colposcopy referral population (except for HSIL).

Moreover, the performances of the combined markers for predicting CIN2+ in different subgroups are shown in Table 3. Of the 606 cases with HPV16/18+ cases in the HPV genotyping test, the sensitivity and specificity of p16+ combined with $\mathrm{Ki}-67+$ for predicting CIN2+ were $67.7 \%$ and $87.6 \%$, respectively, whereas those of $\mathrm{p} 16+$ combined with YAP1+ and/or RELA+ (p16+, YAP1+/RELA+) were 69.2\% $(p=0.850)$ and $91.2 \%(p=0.326)$, respectively. Of the 339 cases that were diagnosed with other $\mathrm{HPV}+$ combined with ASCUS, the combination of p16+, YAP1+/RELA+ produced a better performance for predicting CIN2+, which had better sensitivity $(86.7 \%$ vs $52.2 \%, \mathrm{p}=0.028)$ and considerable specificity $(86.7 \%$ vs $90.0 \%, \mathrm{p}=0.486)$ than $\mathrm{p} 16+$ combined with $\mathrm{Ki}-67+$. The positive predictive value (PPV) and negative predictive value (NPV) also showed in Tables 2 and 3. Moreover, the RELA+ provided the Max AUC area (0.902, 0.837 and 0.875 ) in the colposcopy referral population (except for HSIL) (Figure 3A, Supplementary S1), HPV16/ 18+ group (Figure 3B, Supplementary S2) and LSIL group (Figure 3D, Supplementary S4), respectively. The (p16+, YAP1+/RELA+) provided the Max AUC area (0.847) in 
Table 2 The Positive Rate of PI6, Ki-67, SMAD3, YAPI and RELA Expression and Association with Biopsy CIN2+ in the Enrolled Patients

\begin{tabular}{|l|c|c|c|c|c|c|}
\hline & Positive Rate n (\%) & Sen (\%) & Sep (\%) & PPV (\%) & NPV (\%) & Youden's Index (\%) \\
\hline Pl6+, Ki-67+ & $285(23.9)$ & 62.1 & 89.5 & 67.4 & 87.1 & \\
Pl6+, RELA+ & $255(21.4)$ & 63.1 & 93.2 & 76.5 & 87.9 & 51.6 \\
Pl6+, YAPI+ & $243(20.4)$ & 61.2 & 93.9 & 77.8 & 87.4 & 56.3 \\
PI6+, SMAD3+ & $162(13.6)$ & 35.0 & 93.8 & 66.7 & 80.5 & 55.1 \\
PI6+, YAPI+/RELA+ & $312(26.1)$ & 70.9 & 89.5 & 70.2 & 89.8 & 28.8 \\
PI6+, YAPI+/SMAD3+/RELA+ & $345(28.9)$ & 72.8 & 86.4 & 65.2 & 90.1 & 6.4 \\
\hline
\end{tabular}

Abbreviations: Sen, sensitivity; Sep, specificity; PPV, positive predictive value; NPV, negative predictive value.

the other HPV+ combined with ASCUS group (Figure 3C, Supplementary S3).

The follow-up interval for $711 / 885$ cases which were diagnosis of NILM and CIN1 ranged from 36 to 60 months, contained 93 cases of (p16+, YAP1+/RELA) and 618 cases of others except for (p16+, YAP1+/RELA+). During the follow-up period, 45 cases were developed to CIN2+ (Supplementary S5). 38, 21, 34, 18, 17, 15, 32, 14, 15,37 and 37 of the 45 cases were positive for $\mathrm{p} 16+, \mathrm{Ki}-$ 67+, RELA+, YAP1+, SMAD3+, (p16+, Ki-67+), (p16+, RELA+), (p16+, YAP1+), (p16+, SMAD3+), (p16+, YAP1 $+/ \mathrm{RELA}+)$ and (p16+, YAP1+/SMAD3+/RELA+) at the initial IHC staining. The (p16+, YAP1+/RELA + ) provided the Max AUC area (0.878) for CIN2+ predicting of NILM and CIN1 group in the colposcopy referral population (except for HSIL) (Figure 3E, Supplementary S6).

\section{Discussion}

In the past 30 years, as a result of the extensive development of cervical cancer screening, the incidence of cervical cancer has dropped by more than $50 \%$ in the United States, and the rate of mortality has also decreased. ${ }^{25}$ However, in developing countries, the incidence of cervical cancer is approximately 4-6 times higher than that of developed countries. ${ }^{1}$ There are still many limitations related to cervical cancer screening technologies and strategies. $^{26}$ The independent use of cytology or HPV tests showed poor sensitivity and specificity for the detection of underlying CIN2+, resulting in unnecessary and invasive diagnoses. ${ }^{27}$ In 2015 , the updated cervical cancer screening guidelines of the American Society of Colposcopy \& Cervical Pathology (ASCCP) indicated that the optimal screening methods are co-testing for women aged $30-65$ as this simultaneously increases the sensitivity and specificity of cervical cancer screening. ${ }^{28}$ However, studies have shown that although co-testing effectively improved the effectiveness of cervical cancer screening, 60-80\% of patients except for cytology HSIL who underwent colposcopy did not receive benefits. ${ }^{29-31}$ In the present study, the rate of $\mathrm{CIN} 2+$ in the population who were triaged to colposcopy ranged from $18.1 \%$ to $40 \%$ according to HPV genotyping and liquid-based cytology (Table 1, except for HSIL). Despite the increasing coverage of HPV vaccines, screening will remain necessary for decades to control cervical cancer. Therefore, it is imperative to explore effective triage strategies to reduce misdiagnoses and unnecessary invasive examinations.

The triage strategies that are currently evaluated include p16/Ki-67 immunocytochemistry, HPV E6/E7 mRNA testing, host methylation, and viral methylation testing. In the 2014 edition of the Bethesda System for Reporting Cervical Cytology, immunocytochemistry was recommended as an adjuvant test for cytological diagnosis. ${ }^{32}$ In recent years, many studies have investigated the application of cervical immunocytochemical staining in clinics. A study performed in Italy showed that relatively better sensitivity $(79.7 \%)$ and specificity $(73.5 \%)$ were achieved by dual staining in detecting CIN2+ lesions. $^{33}$ Wentzensen et al $^{16}$ examined 625 patients who were referred to colposcopy and showed that dual staining exhibited a sensitivity of $86.4 \%(81.5-$ $90.2 \%$ ) and a specificity of $59.5 \%$ (54.2-64.5\%) for detecting CIN2+. However, for patients with HR-HPV+ and concurrent ASCUS/LSIL, p16/Ki67 dual staining exhibited high sensitivity $(90.6 \%, 73.8-97.5)$ but poor specificity $(48.6 \%, 43.5-53.9)$ for detecting CIN3+. In addition, the VUSA Screen study showed that the sensitivity of p16/Ki67 dual staining for patients with HPV (+) combined with ASCUS was significantly higher than that for patients with HPV (+) TCT (-) (91\% vs 68.8\%), although its specificity was poor $(30.6 \%$ vs $72.8 \%){ }^{34}$ Moreover, data from China showed that the sensitivity of p16/Ki67 dual staining was not superior to cytology $(92.7 \%$ vs $94.5 \%)$, nor the specificity $(52.7 \%$ vs $53.5 \%)$ 


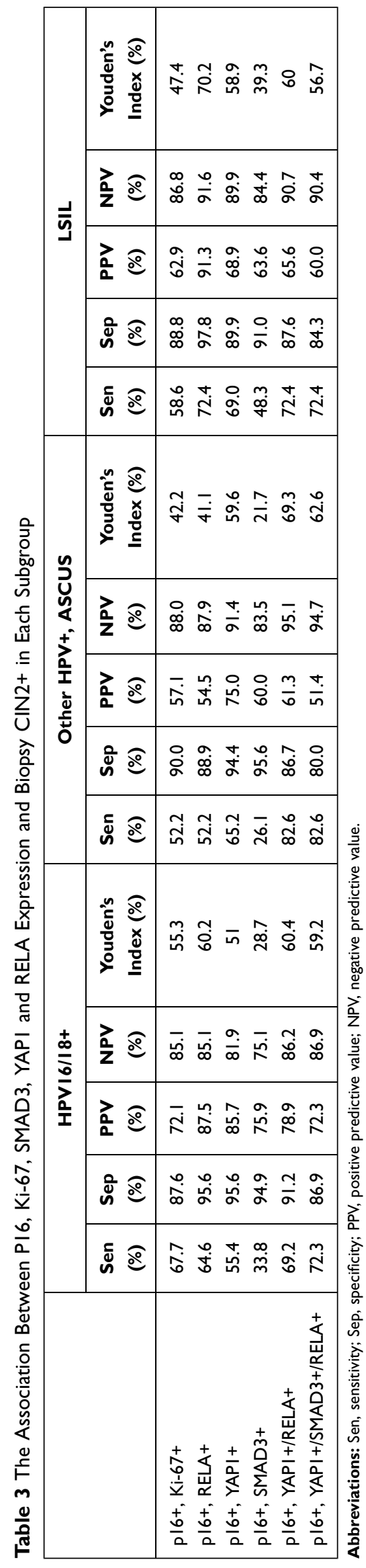

in patients with HR-HPV+. For patients with ASCUS/ LSIL, the specificity was increased only $11 \%(66.4 \%$ vs $55.8 \%$ ) of p16/Ki67 dual staining for detecting CIN2+, although the colposcopy referral rate was reduced $10 \%$ $(43.8 \%$ vs $53.1 \%) .{ }^{18}$ Taken together, these data indicate that although p16/Ki67 dual staining reduces the colposcopy referral rate, its sensitivity and specificity need to be enhanced. Therefore, more sensitive and specific biomarkers should be investigated to improve the accuracy of cervical cancer screening and reduce unnecessary invasive examinations.

Galgano et al reported that p16 immunostaining, performed using the strongest staining as the cutpoint, was $86.7 \%$ sensitive and $82.8 \%$ specific for detecting CIN $2+.{ }^{35}$ A review of p16 immunohistochemistry in cervical lesions also showed that the positive rates of p16 ranged from $0 \%$ to $37.5 \%, 0 \%$ to $100 \%$, and $61.5 \%$ to $100 \%$ in the negative, LSIL and HSIL groups, respectively. While the positive rates of $\mathrm{Ki}-67$ ranged from $0 \%$ to $81.1 \%, 12.5 \%$ to $100 \%$, and $42.3 \%$ to $100 \%$ in each group. ${ }^{36}$ It has been suggested that the different positivity rates observed across studies may be due to multiple factors, such as different staining conditions, clones, patient populations, and lesion grading thresholds. ${ }^{37}$ In the present study, the correlations between the expression levels of p16, Ki-67, and three shape-correlated genes (SMAD3, YAP1 and RELA) and CIN2+ lesions were evaluated by IHC. The sensitivity and specificity of p 16 were $82.5 \%$ and $70.5 \%$, respectively, for detecting CIN2+, consistent with the literature. RELA and a combination consisting of p16, YAP1 and RELA had better sensitivity and specificity than was achieved by p16 alone for detecting CIN2+ in different subgroups. Notably, 45 cases that showed $<\mathrm{CIN} 2$ at the initial biopsy were diagnosed as CIN2+ during the follow-up period, and 37 of these 45 cases were positive for p16+, RELA+/YAP1+ at the initial IHC staining, which also provided a Max AUC area for predicting CIN2+. Hence, we suggest that a combination consisting of p16, RELA and YAP1 may represent a biomarker for underlying CIN2+ in cervical screening. The current study also has several limitations. First, there may be inevitable errors in the data reading of immunohistochemistry in the present study. Second, this study is a retrospective study, there is the possibility of degradation of frozen tissue protein, which affects the authenticity of the results. Most importantly, further cytological staining experiment is necessary to evaluate the clinical feasibility. 


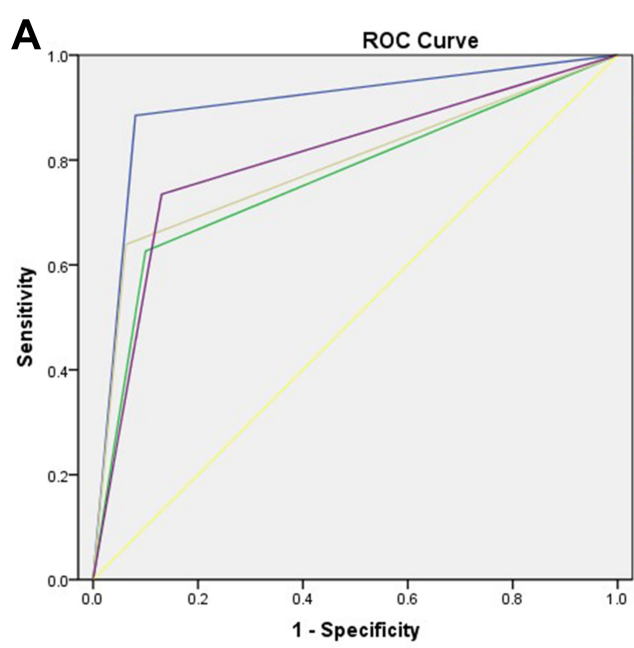

C

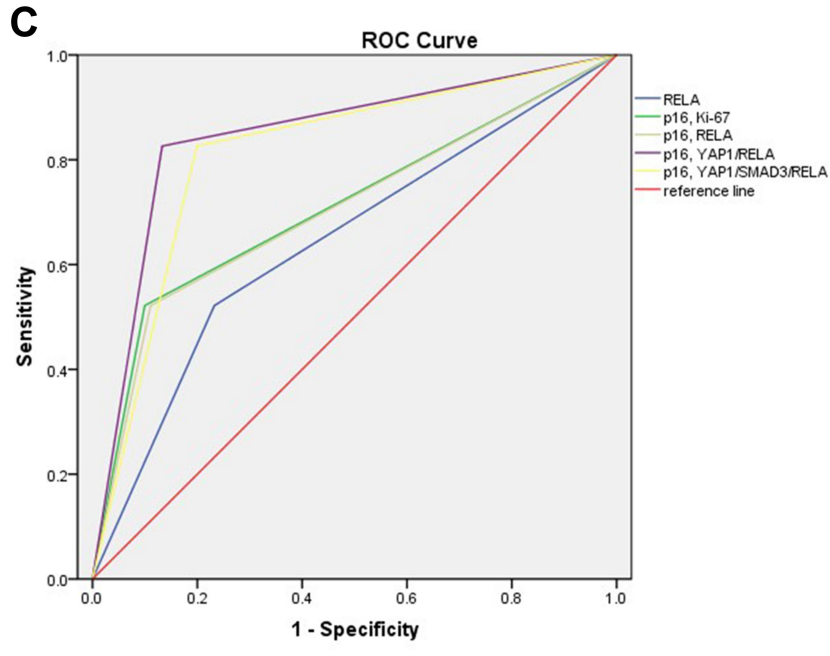

B

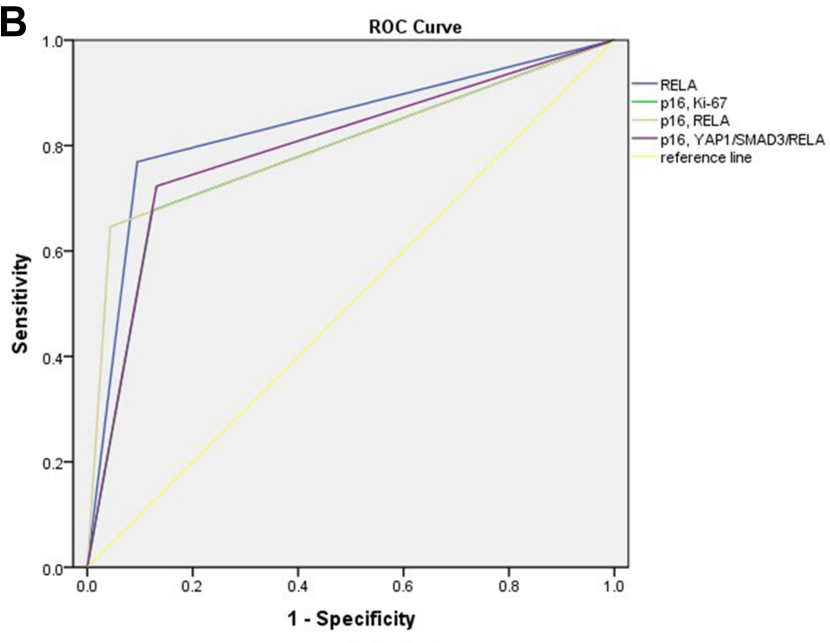

D

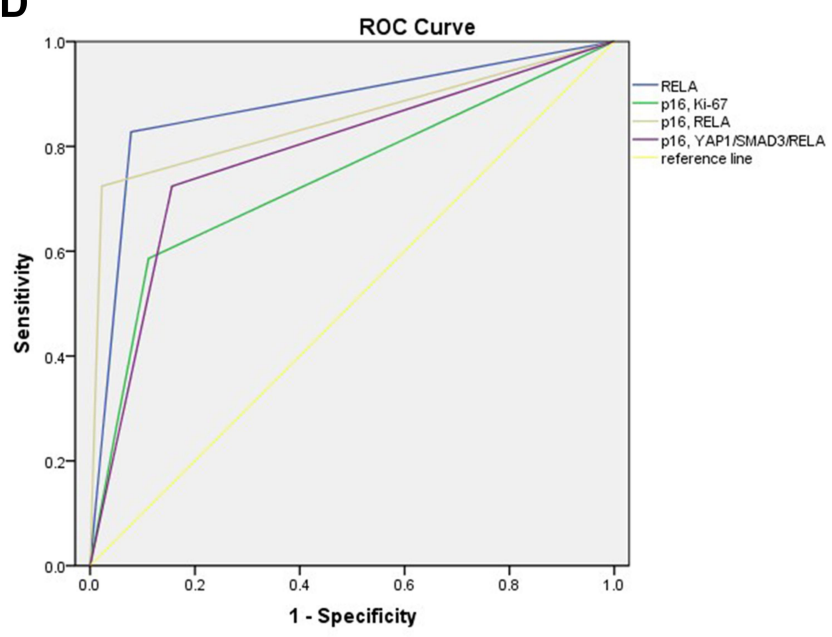

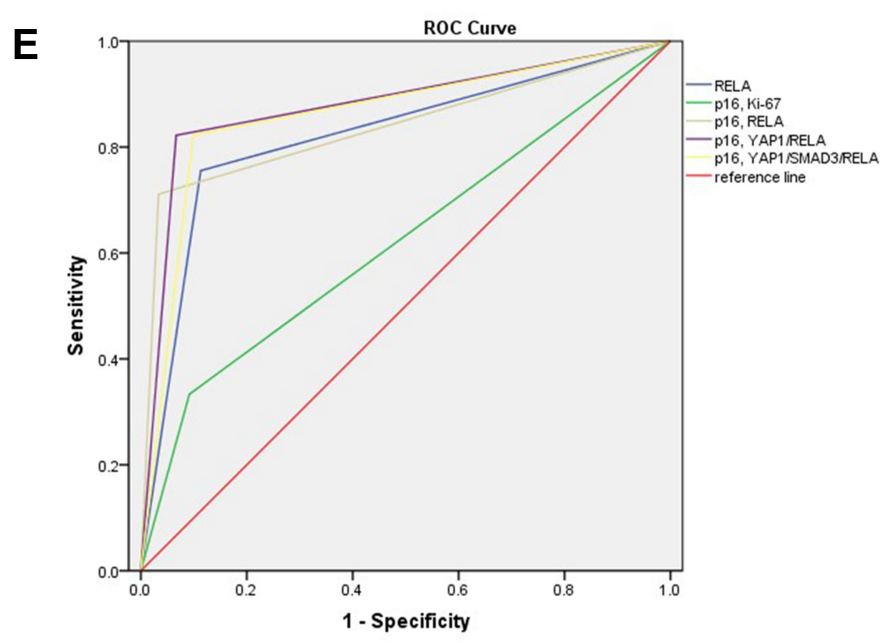

Figure 3 The AUC for p 16, Ki-67, SMAD3, YAPI and RELA in different groups. (A) the colposcopy referral population (except for HSIL). (B) HPVI6/18+ group. (C) other $\mathrm{HPV}+$ combined with ASCUS group. (D) LSIL group. (E) CIN2+ predicting during follow-up.

Our aim is to investigate more disease-specific molecular markers which can provide an improved triage strategy for colposcopy after co-testing. Our team has also conducted a project to evaluate the immunocytochemical staining performance of correlative markers in screening populations. We expected to present better results in the future. Cytology HSIL showed a high sensitivity and specificity for detecting CIN2+, therefore we did not focus on this subgroup. 


\section{Conclusion}

Our study shows that the rate of CIN2+ ranged from $18.1 \%$ to $40 \%$ in a population of patients who were triaged to colposcopy according to HPV genotyping and liquidbased cytology (except for cytology HSIL). RELA and a combination consisting of p16 and RELA/YAP1 IHC achieved superior sensitivity and specificity for detecting morphologically CIN2+ lesions in the colposcopy referral population. However, further studies will be needed to evaluate the immunocytochemical staining performance of correlative markers.

\section{Ethical Approval and Consent Statement}

All procedures performed in studies involving human participants were in accordance with the ethical standards of the institutional and national research committee and with the 1964 Helsinki declaration and its later amendments. The name and affiliation of the ethics committee that approved this study: The institutional ethics committee of Peking Union Medical College Hospital, CAMS Chinese Academy of Medical Sciences, No. S-T642 2014. Informed consent was obtained from individual participants included in the study.

\section{Funding}

This study was funded by the Beijing Science and Technology Plan Project [D151100001915004] (Keng Shen) and [CAMS Innovation Fund for Medical Sciences (CIFMS) CAMS-2017-I2M-1-002] (Keng Shen).

\section{Disclosure}

The authors report no conflicts of interest in this work.

\section{References}

1. Bray F, Ferlay J, Soerjomataram I, Siegel RL, Torre LA, Jemal A. Global cancer statistics 2018: GLOBOCAN estimates of incidence and mortality worldwide for 36 cancers in 185 countries. CA Cancer J Clin. 2018;68(6):394-424. doi:10.3322/caac.21492

2. Richart RM. Causes and management of cervical intraepithelial neoplasia. Cancer. 1987;60(8 Suppl):1951-1959.

3. Ferlay J, Shin HR, Bray F, Forman D, Mathers C, Parkin DM. Estimates of worldwide burden of cancer in 2008: GLOBOCAN 2008. Int J Cancer. 2010;127(12):2893-2917. doi:10.1002/ijc.25516

4. van der Aa MA, Pukkala E, Coebergh JW, Anttila A, Siesling S. Mass screening programmes and trends in cervical cancer in Finland and the Netherlands. Int J Cancer. 2008;122(8):1854-1858. doi:10.1002/ijc.23 276

5. Committee on Practice Bulletins - Gynecology. Practice bulletin No. 168: cervical cancer screening and prevention. Obstet Gynecol. 2016;128(4):111-130. doi:10.1097/AOG.0000000000001708
6. Murphy J, Kennedy EB, Dunn S, et al. HPV testing in primary cervical screening: a systematic review and meta-analysis. J Obstet Gynaecol Can. 2012;34(5):443-452. doi:10.1016/S1701-2163(16)35241-0

7. Graham SV. The human papillomavirus replication cycle, and its links to cancer progression: a comprehensive review. Clin Sci (Lond). 2017:131(17):2201-2221.

8. de Carvalho SH, Rosa DAN, Santos ALF, de Medeiros APP. Description of referrals for colposcopy in a hospital in Brazil. Rev Bras de Ginecol e Obstet. 2020;42(3):140-145. doi:10.1055/s-0040-1708886

9. Loopik DL, Koenjer LM, Siebers AG, Melchers WJG, Bekkers RLM. Benefit and burden in the Dutch cytology-based vs high-risk human papillomavirus-based cervical cancer screening program. Am J Obstet Gynecol. 2020.

10. Wentzensen N, Walker J, Smith K, et al. A prospective study of risk-based colposcopy demonstrates improved detection of cervical precancers. Am J Obstet Gynecol. 2018;218(6):604.e601-604.e608. doi:10.1016/j.ajog.2018.02.009

11. Torres-Ibarra L, Cuzick J, Lorincz AT, et al. Comparison of HPV-16 and HPV-18 genotyping and cytological testing as triage testing within human papillomavirus-based screening in Mexico. JAMA Netw Open. 2019;2(11):e1915781. doi:10.1001/jamanetworkopen.2019.15781

12. Darragh TM, Colgan TJ, Thomas Cox J, et al. The lower anogenital squamous terminology standardization project for HPV-associated lesions: background and consensus recommendations from the college of American pathologists and the American society for colposcopy and cervical pathology. Int J Gynecol Cancer. 2013;32 (1):76-115. doi:10.1097/PGP.0b013e31826916c7

13. Ikenberg H, Bergeron C, Schmidt D, et al. Screening for cervical cancer precursors with $\mathrm{p} 16 / \mathrm{Ki}-67$ dual-stained cytology: results of the PALMS Study. J Natl Cancer Inst. 2013;105(20):1550-1557. doi:10. 1093/jnci/djt235

14. Wright TC Jr, Behrens CM, Ranger-Moore J, et al. Triaging HPV-positive women with p16/Ki-67 dual-stained cytology: results from a sub-study nested into the ATHENA trial. Gynecol Oncol. 2017;144(1):51-56. doi:10.1016/j.ygyno.2016.10.031

15. Bergeron C, Ikenberg H, Sideri M, et al. Prospective evaluation of p16/Ki-67 dual-stained cytology for managing women with abnormal papanicolaou cytology: PALMS Study results. Cancer Cytopathol. 2015;123(6):373-381. doi:10.1002/cncy.21542

16. Wentzensen N, Schwartz L, Zuna RE, et al. Performance of p16/ $\mathrm{Ki}-67$ immunostaining to detect cervical cancer precursors in a colposcopy referral population. Clin Cancer Res. 2012;18(15): 4154-4162. doi:10.1158/1078-0432.CCR-12-0270

17. Uijterwaal MH, Polman NJ, Witte BI, et al. Triaging HPV-positive women with normal cytology by p16/Ki-67 dual-stained cytology testing: baseline and longitudinal data. Int J Cancer. 2015;136 (10):2361-2368. doi:10.1002/ijc.29290

18. Yu LL, Chen W, Lei XQ, et al. Evaluation of p16/Ki-67 dual staining in detection of cervical precancer and cancers: a multicenter study in China. Oncotarget. 2016;7(16):21181-21189. doi:10.18632/oncotarget. 8307

19. Perkins RB, Guido RS, Castle PE, et al. 2019 ASCCP risk-based management consensus guidelines for abnormal cervical cancer screening tests and cancer precursors. J Low Genit Tract Dis. 2020;24(2):102-131. doi:10.1097/LGT.0000000000000525

20. Sailem HZ, Bakal C. Identification of clinically predictive metagenes that encode components of a network coupling cell shape to transcription by image-omics. Genome Res. 2017;27(2):196-207. doi:10. 1101/gr.202028.115

21. Zhou Q, Han LR, Zhou YX, Li Y. MiR-195 suppresses cervical cancer migration and invasion through targeting Smad3. Int J Gynecol Cancer. 2016;26(5):817-824. doi:10.1097/IGC.00000 00000000686

22. Xiao H, Wu L, Zheng H, et al. Expression of yes-associated protein in cervical squamous epithelium lesions. Int $J$ Gynecol Cancer. 2014;24(9):1575-1582. doi:10.1097/IGC.0000000000000259 
23. Alzahrani F, Clattenburg L, Muruganandan S, et al. The Hippo component YAP localizes in the nucleus of human papilloma virus positive oropharyngeal squamous cell carcinoma. J Otolaryngol. 2017;46(1):15.

24. Nair A, Venkatraman M, Maliekal TT, Nair B, Karunagaran D. NFkappaB is constitutively activated in high-grade squamous intraepithelial lesions and squamous cell carcinomas of the human uterine cervix. Oncogene. 2003;22(1):50-58. doi:10.1038/sj.onc.1206043

25. Siegel RL, Miller KD, Jemal A. Cancer statistics, 2016. CA Cancer J Clin. 2016;66(1):7-30. doi:10.3322/caac.21332

26. Saxena U, Sauvaget C, Sankaranarayanan R. Evidence-based screening, early diagnosis and treatment strategy of cervical cancer for national policy in low-resource countries: example of India. Asian Pac J Cancer Prev. 2012;13(4):1699-1703. doi:10.7314/APJCP.20 12.13.4.1699

27. Wright TC Jr., Stoler MH, Behrens CM, Sharma A, Sharma K, Apple R. Interlaboratory variation in the performance of liquid-based cytology: insights from the ATHENA trial. Int $J$ Cancer. 2014;134(8):1835-1843. doi:10.1002/ijc.28514

28. Huh WK, Ault KA, Chelmow D, et al. Use of primary high-risk human papillomavirus testing for cervical cancer screening: interim clinical guidance. Gynecol Oncol. 2015;136(2):178-182. doi:10.10 16/j.ygyno.2014.12.022

29. Wentzensen N, Schiffman M, Palmer T, Arbyn M. Triage of HPV positive women in cervical cancer screening. J Clin Virol. 2016;76 (Suppl 1):S49-s55. doi:10.1016/j.jcv.2015.11.015

30. Hillemanns P, Soergel P, Hertel H, Jentschke M. Epidemiology and early detection of cervical cancer. Oncol Res Treat. 2016;39 (9):501-506. doi:10.1159/000448385
31. Ordi J, Sagasta A, Munmany M, Rodriguez-Carunchio L, Torne A, Del Pino M. Usefulness of p16/Ki67 immunostaining in the triage of women referred to colposcopy. Cancer Cytopathol. 2014;122 (3):227-235. doi:10.1002/cncy.21366

32. Solomon D, Nayar R. The Bethesda System for Reporting Cervical Cytology: Definitions, Criteria, and Explanatory Notes. Springer Verlag; 2004.

33. Dona MG, Vocaturo A, Giuliani M, et al. p16/Ki-67 dual staining in cervico-vaginal cytology: correlation with histology, human papillomavirus detection and genotyping in women undergoing colposcopy. Gynecol Oncol. 2012;126(2):198-202. doi:10.1016/j.ygyno.2012.05.004

34. Uijterwaal MH, Witte BI, Van Kemenade FJ, et al. Triaging borderline/mild dyskaryotic Pap cytology with p16/Ki-67 dual-stained cytology testing: cross-sectional and longitudinal outcome study. $\mathrm{Br}$ $J$ Cancer. 2014;110(6):1579-1586. doi:10.1038/bjc.2014.34

35. Galgano MT, Castle PE, Atkins KA, Brix WK, Nassau SR, Stoler MH. Using biomarkers as objective standards in the diagnosis of cervical biopsies. Am J Surg Pathol. 2010;34(8):1077-1087. doi:10.1097/PAS.0b013e3181e8b2c4

36. Silva DC, Goncalves AK, Cobucci RN, Mendonca RC, Lima PH, Cavalcanti GJ. Immunohistochemical expression of p16, Ki-67 and p53 in cervical lesions - A systematic review. Pathol Res Pract. 2017;213(7):723-729. doi:10.1016/j.prp.2017.03.003

37. Paquette C, Mills AM, Stoler MH. Predictive value of cytokeratin 7 immunohistochemistry in cervical low-grade squamous intraepithelial lesion as a marker for risk of progression to a high-grade lesion. $\mathrm{Am}$ J Surg Pathol. 2016;40(2):236-243. doi:10.1097/PAS.000000000000 0548

\section{Publish your work in this journal}

OncoTargets and Therapy is an international, peer-reviewed, open access journal focusing on the pathological basis of all cancers, potential targets for therapy and treatment protocols employed to improve the management of cancer patients. The journal also focuses on the impact of management programs and new therapeutic agents and protocols on patient perspectives such as quality of life, adherence and satisfaction. The manuscript management system is completely online and includes a very quick and fair peer-review system, which is all easy to use. Visit http://www.dovepress.com/ testimonials.php to read real quotes from published authors 